\title{
OBSERVATIONS OF GAMMA-RAY BURSTS
}

\author{
G.J. Fishman \\ Space Sciences Laboratory \\ NASAMarshall Space Flight Center \\ Huntsville, AL 35812 USA
}

\begin{abstract}
Some basic observed properties of gamma-ray bursts are reviewed. Although some properties were known 25 years ago, new and more detailed observations have been made by the Compton Observatory in the past three years. The new observation with the greatest impact has been the observed isotropic distribution of bursts along with a deficiency of weak bursts which would be expected from a homogeneous burst distribution. This is not compatible with any known Galactic population of objects. Gamma-ray bursts show an enormous variety of burst morphologies and a wide spread in burst durations. The spectra of gamma-ray bursts are characterized by rapid variations and peak power which is almost entirely in the gamma-ray energy range. Delayed gamma-ray burst photons extending to $\mathrm{GeV}$ energies have been detected for the first time. A time dilation effect has also been reported to be observed in gamma-ray bursts. The observation of a gamma-ray burst counterpart in another wavelength region has yet to be made.
\end{abstract}

\section{Introduction}

It is over 25 years since the discovery of gamma-ray bursts, and their origin appears as elusive as ever. The observed isotropy and inhomogeneity of these objects represent a distribution unlike any other known galactic objects. Over a hundred theories on their origin have now been cataloged (Nemiroff 1994). These models cover distance scales from the local Oort cloud to cosmological distances. Never before in modern astronomy has there been so great an uncertainty in the distance to a class of objects, as well as a similar level of uncertainty about the basic nature of the source and the emission mechanism. Whatever the distance scale and the source of the bursts turn out to be, it will most likely represent a new class of objects, processes and/or emission mechanism. Thus, there is simultaneously a frustration and an excitement in the field.

This lecture summarizes the basic observed properties of gamma-ray bursts, primarily their temporal and spectral characteristics and their distribution. Two other papers in these proceedings address the statistical studies of gamma-ray bursts (Atteia 1994) and the astrophysical considerations surrounding the wide variety of gamma-ray burst models (Hartmann 1994) 

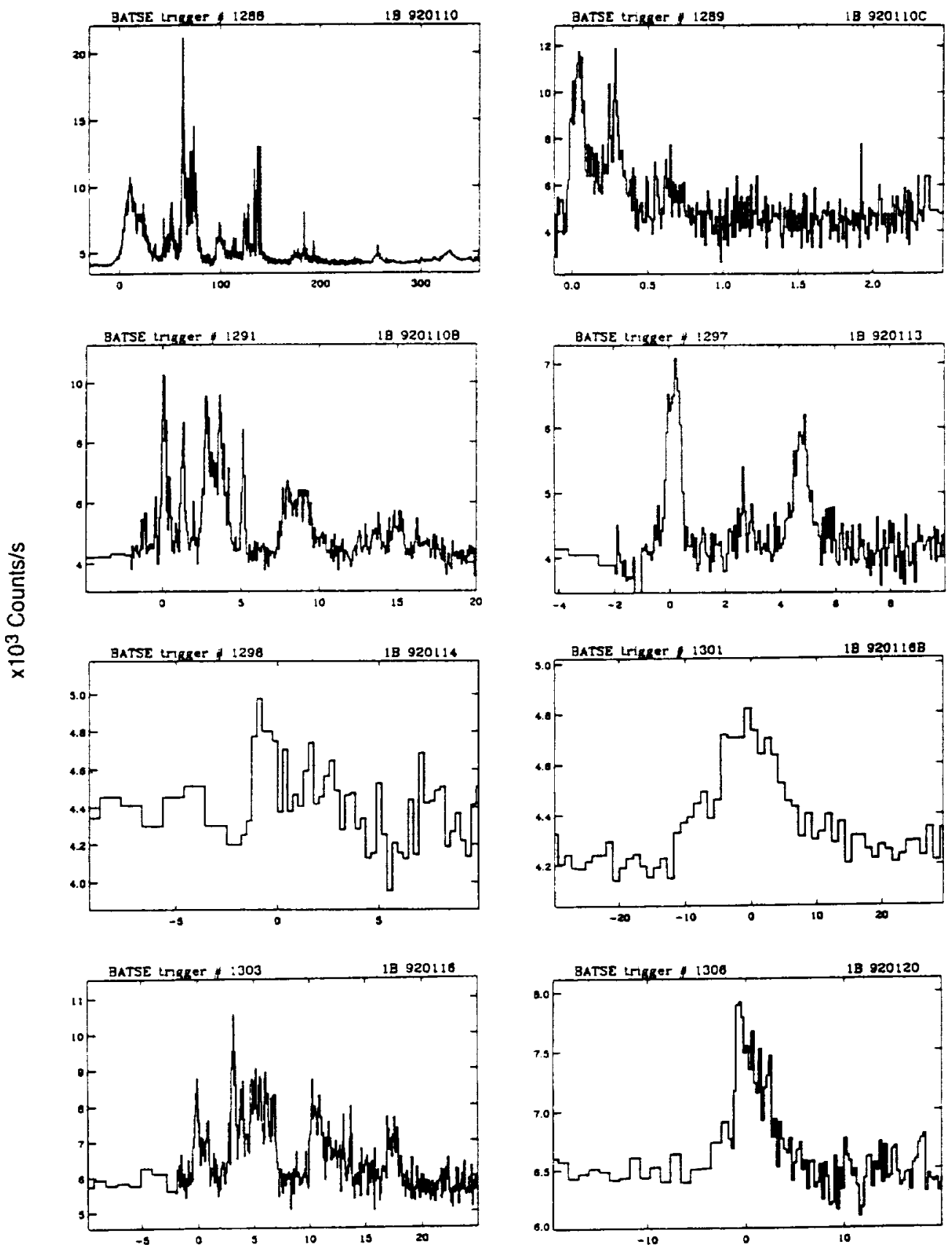

Seconds (Rel. to Trigger)

Figure 1. A sample of eight gamma-ray bursts for the First BATSE Catalog (Fishman et al. 1994), showing the extreme range of burst time profiles and durations 
Considerable observational progress has been made in the past few years as more sensitive space-borne detectors have become available. Most of the observations in this paper were made by the Burst and Transient Source Experiment (BATSE) on the Compton Gamma-Ray Observatory. While many of the observational results are relatively straight-forward, some of the properties and interpretations of ensembles of bursts are the subject of analyses that are often debatable. Comprehensive sets of papers of the more recent observational results can be found in conference proceedings that have been published in the past three years (Paciesas and Fishman 1992; Friedlander, Gehrels and Macomb 1993; Fishman, Brainerd and Hurley 1994), as well as in the astrophysical scientific literature. Hartmann (1994) also provides a detailed list of gamma-ray burst reviews and Hurley (1994) has made available a comprehensive bibliography of the gamma-ray burst literature.

\section{Time Profiles}

Perhaps the most striking features of the time profiles of gamma-ray bursts are their morphological diversity and the large range of burst durations. Coupled with this diversity is the general inability to place many gamma-ray bursts into well-defined classifications. Although this was known from previous experiments, the large sensitive area of the BATSE detectors shows this well in the First BATSE Gamma-ray Burst Catalog (Fishman et al. 1994). Examples of extreme differences in burst morphologies and durations are shown in a sample page from the First BATSE Burst Catalog (Figure 1)

The durations of gamma-ray bursts range from about $10 \mathrm{~ms}$ to over $1000 \mathrm{~s}$ in the energy range in which most bursts are observed. However, recent EGRET observations show high energy $(>100 \mathrm{MeV})$ emission over 90 minutes after the burst trigger (EGRET Team, presented at American Physical Society Meeting, Crystal City, Va, USA, April 1994; yet unpublished). Sub-millisecond structure has been detected in at least one burst (Bhat et al. 1992). Weaker bursts show the same diversity as the stronger bursts even though the temporal variations are of lower statistical significance (Lestrade 1994). A cursory examination of burst profiles indicates that some are chaotic and spiky with large fluctuations on all timescales, while others show rather simple structures with few peaks. However, some bursts are seen with both characteristics present within the same burst. No periodic structures have been seen from gamma-ray bursts.

The duration of a gamma-ray burst is difficult to quantify since this quantity is dependent on the intensity and background and somewhat dependent upon the time resolution of the experiment. The BATSE group has settled on a T-90 measure, the time over which $90 \%$ of the burst fluence is detected. A recent compilation of the distribution of durations is shown in Figure 2 (Fishman et al. 1994). A bi-modality is seen in the logarithmic distribution with broad, un-resolved peaks at about $0.3 \mathrm{~s}$ and $20 \mathrm{~s}$ and a minimum at around $2 s$. The fall-off at short durations is partially due to an instrumental bias, since the minimum time scale over which the BATSE experiment can trigger on bursts is $64 \mathrm{~ms}$. The shorter bursts are also seen to have harder spectra, as measured by a hardness ratio (Kouveliotou et al. 1994). Another general property of the gamma-ray bursts time 


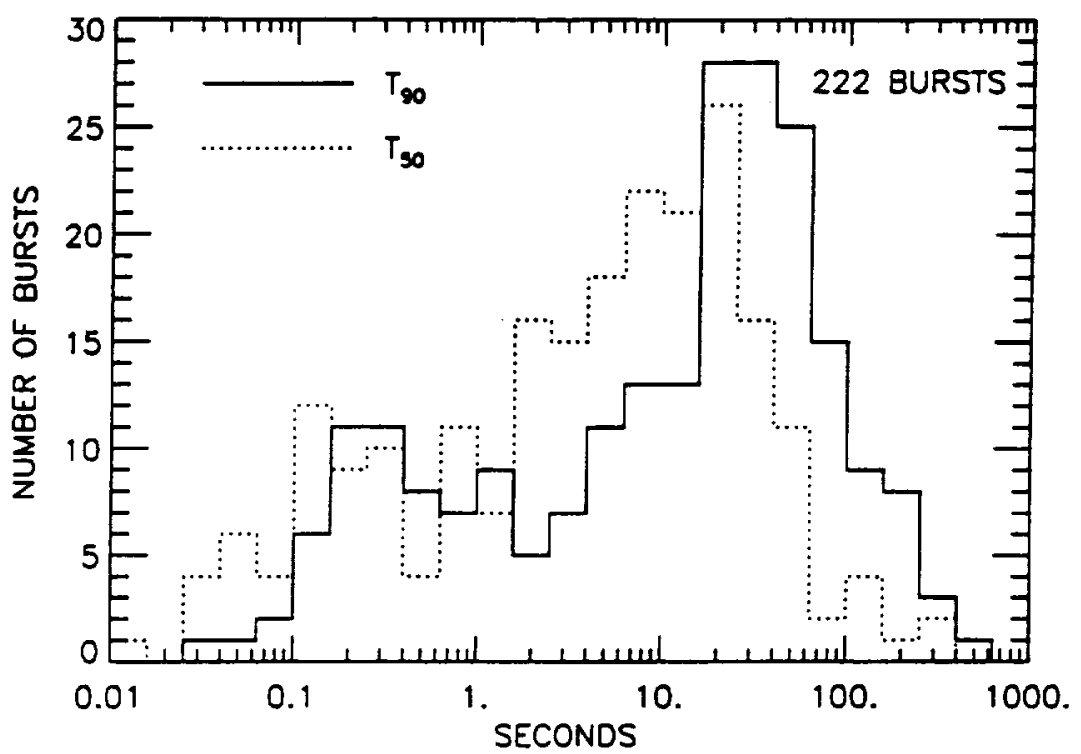

Figure 2. The duration distribution of gamma-ray bursts from the First BATSE Burst Catalog (Fishman et al., 1994).

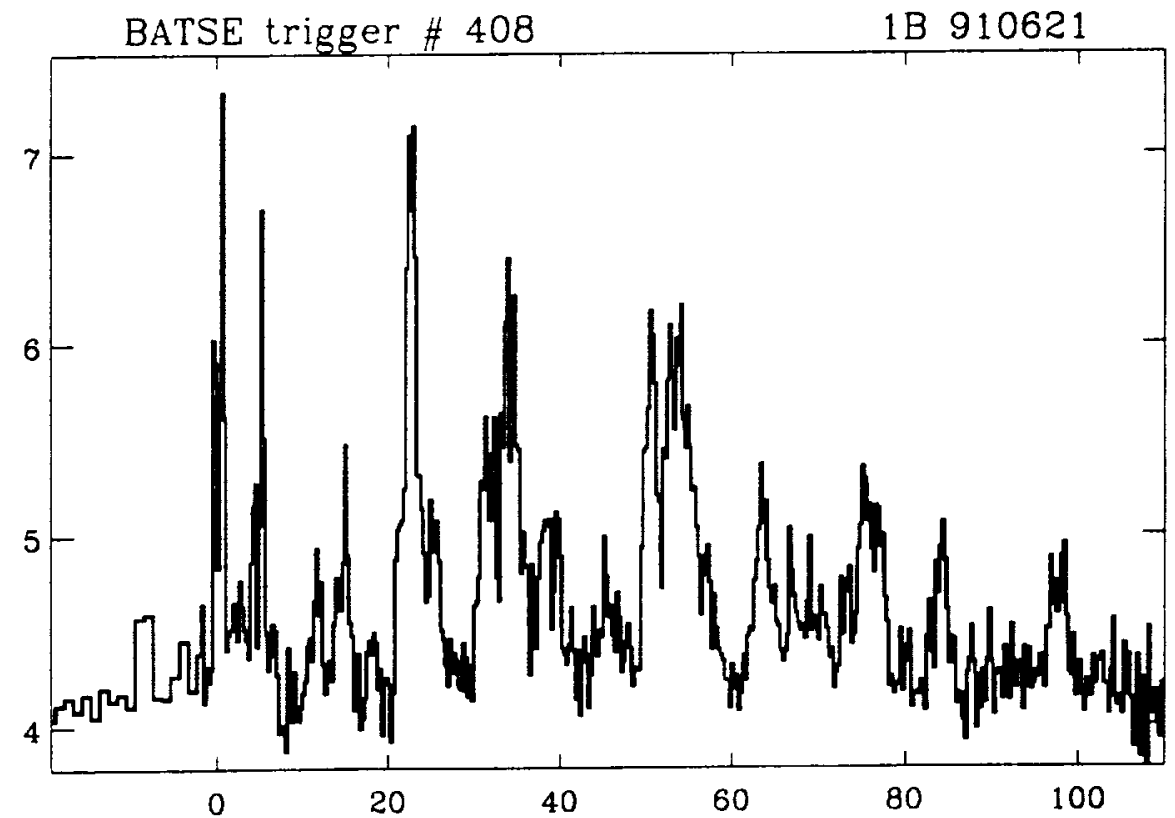

Figure 3. Gamma-ray burst GB 910621 from the BATSE catalog. This is an example of a burst that shows no asymmetry in its time profile. 
profiles is that they tend to have shorter rise-times and fall-times (sharper spikes) at higher energies. Most bursts also show an asymmetry, with shorter leading edges than trailing edges. This has been quantified by Link, Epstein and Priedhorsky (1993) and by Nemiroff et al. (1994). These authors use this observation as an argument for an explosive event rather than a sweeping, beamed event, since the later would, in general, not show such an asymmetry. However, some bursts show no asymmetry (cf. Figure 3), even when high counting rates would permit such measurements to be made. Other analyses have used a variety of temporal parameters and constructs (for example see Lestrade 1994; Brock, et al. 1994; Meredith et al. 1994) to quantify and characterize gamma-ray burst temporal properties.

A recent analysis of time profiles that could have extraordinary consequences is the observation by Norris et al. (1994) of a systematic widening or stretching of gamma-ray burst time profiles as bursts become weaker. This analysis was performed by artificially weakening the stronger gamma-ray bursts and introducing the appropriate background so that all bursts could be analyzed in a consistent manner. The quantitative analysis of the time profiles was made through the use of wavelets. It is claimed that wavelets are wellsuited to gamma-ray burst analysis because of the apparent random structure and finite duration of the bursts. The observed stretching of the profiles of bursts is consistent with that expected from the effects of time-dilation from bursts at cosmological distances. However, the time dilation observation and its interpretation are not universally accepted. This controversy is progressing as this paper is being written, and several preprints questioning this result are in various stages of preparation.

\section{Spectral Characteristics}

The unique feature of gamma-ray bursts is their high-energy emission: almost all of the power is emitted above $50 \mathrm{keV}$. Some bursts show emission as low as $1 \mathrm{keV}$, but the power is less than 1 or 2 percent of the total power (Yoshida et al. 1989). Most bursts show rather simple continua spectra which appear similar in shape when integrated over the entire burst and when sampled on various timescales within a burst. Figure 4 shows a typical burst spectrum from $0.1 \mathrm{MeV}$ to $10 \mathrm{MeV}$, with the peak power at about $600 \mathrm{keV}$ (Share et al. 1994). The COMPTEL experiment has observed peak power to be in the $\mathrm{MeV}$ energy range (cf. Figure 5; Hanlon et al. 1994). A spectrum from the strong burst GRB 910503, using data from all four GRO experiments is shown in Figure 6 (Schaefer et al. 1994). Spectral shapes which have been fit to burst spectra include broken power laws (Schaefer et al. 1992), log-normal distributions (Pendleton et al. 1994), and exponential spectra with power-law high energy tails (Band et al. 1993). When a single power law is fit to a relatively narrow energy range, such as that measured by the BATSE Large Area Detectors (LAD's), the resulting distribution of power law spectral indices is shown in Figure 7 (Pendleton et al. 1994). Although the spectral shapes of many bursts are similar, the energy at which peak power is emitted changes greatly from burst-to-burst and is seen to rapidly change within a burst. Some significant changes on time scales as short as tens of milliseconds have been observed (cf. Ford et al. 1994). Earlier observations by the gamma-ray spectrometer on the Solar Maximum Mission showed that in many bursts, the 


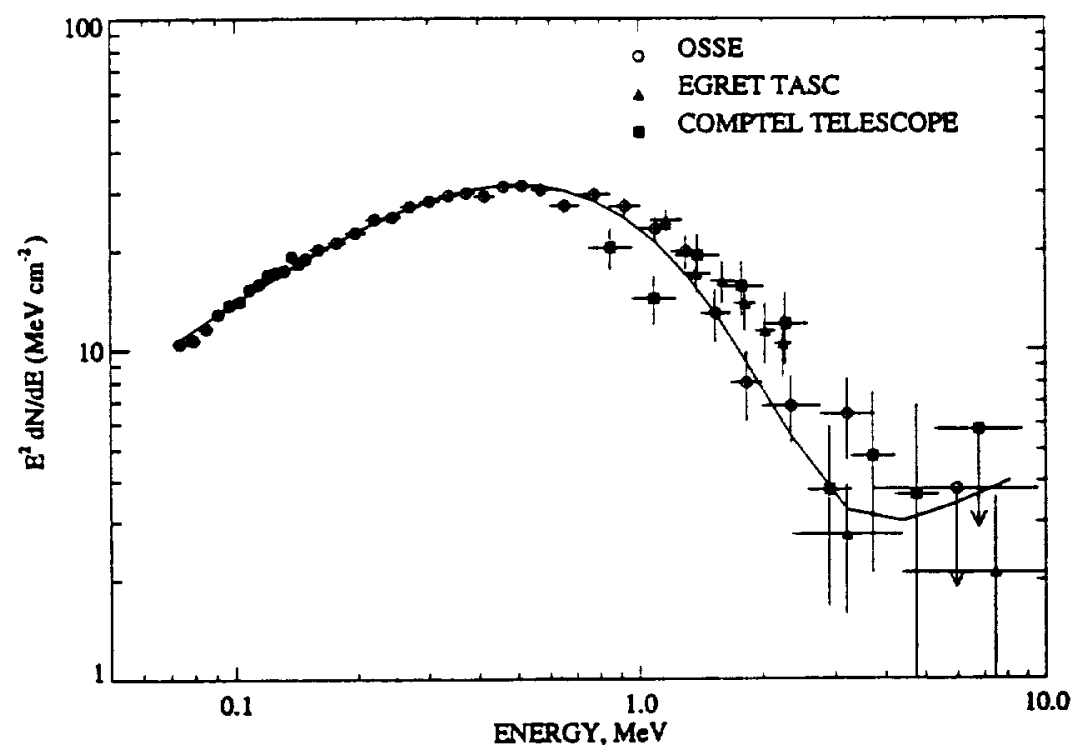

Figure 4. The high energy spectrum from GB 910601, as measured by three of the experiments on the Compton Observatory (Share et al. 1994), integrated over a large portion of the burst. A characteristic broad spectral shape, with peak power of about $0.6 \mathrm{MeV}$ is seen. (The spectral up-turn at high energies is not real.)

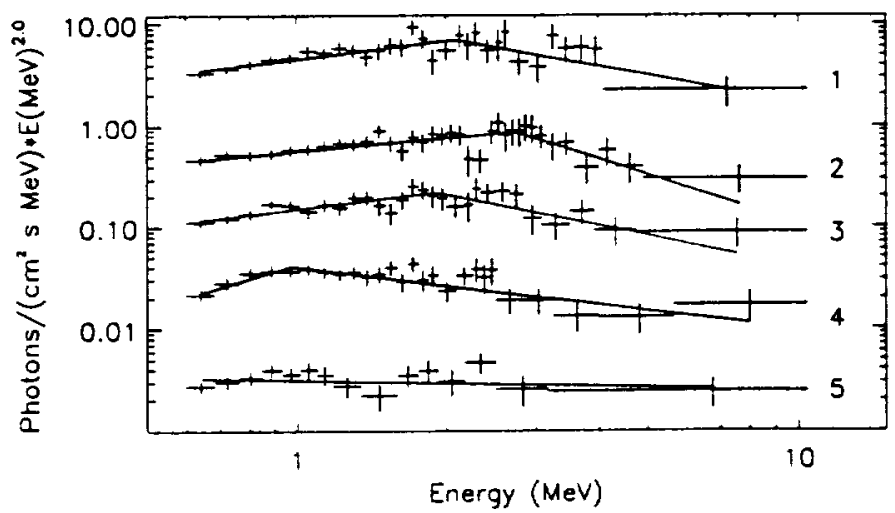

Figure 5. The spectrum of GB 910814 , measured during five intervals of the burst by the COMPTEL experiment on the Compton Observatory (Hanlon et al. 1994). When fit by a broken power-law, the spectra are seen to change over the burst such that the break in the power law correlates with the intensity of the burst. This is seen in most bursts. The break energies seen here are unusually high (cf. Schaefer et al. 1992). 
high energy emission follows the same power law to over $80 \mathrm{MeV}$ (Share et al. 1988). More recently, EGRET has seen significant flux and power into the GeV energy range from several bursts. Many of these high energy photons are delayed with respect to the bulk of the lower energy emission (see Figure 7, from Dingus et al. 1994). A recent observation of EGRET has shown that GeV photons emitted from a gamma-ray burst region are observed over one hour following the burst (EGRET Team; late paper, APS Meeting, Crystal City VA, USA, April 1994; as yet unpublished). There was no observed emission at lower energies from the burst region at that time, as observed by the BATSE experiment. A single $26 \mathrm{GeV}$ photon was recorded from the burst direction which is the highest energy ever recorded from a gamma-ray burst.

Other generalizations can be made with regard to burst continua spectra. Within most (but not all) bursts, there is a hard-to-soft spectral evolution, resulting in the lower energies peaking earlier (Pendleton et al. 1994; Ford et al. 1994). It has also been noted that in general, shorter bursts have harder spectra (Kouveliotou et al. 1993). Pendleton et al. (1994) show some examples of very different continua spectra occurring within the same burst. Superposition of these spectra within a burst can produce "cusps" in the timeintegrated spectra, leading to erroneous indications of spectral features in some gammaray bursts

A search for unambiguous gamma-ray line features with BATSE/GRO has thus far been unable to confirm the earlier reports of spectral line features from gamma-ray bursts. Several recent papers from conference proceedings (Paciesas and Fishman 1992; Fishman, Brainerd and Hurley 1994) have discussed the preliminary BATSE line search analyses and their results. This work is still in progress.

\section{Burst Counterparts}

There is no doubt that a great advance in our understanding of gamma-ray bursts can be attained through successful correlated observations of gamma-ray bursts at other wavelengths. This fact was demonstrated recently by the combined gamma-ray, $x$-ray, optical and radio observations of Soft Gamma-ray Repeaters (SGR's) (Kouveliotou et al. 1994; Murakami et al. 1994; Kulkarni et al. 1994). Within the past three years, there have been major, renewed efforts to find a counterpart to a gamma-ray burst in other wavelength regions as evidenced by either simultaneous emission or afterglow emission. Comprehensive studies of archival plates also have been made. There have been several suggestions for counterparts although the results are often debated. In view of the importance of the implied results, further observational evidence is needed before these results are accepted. Some of the world's most powerful ground-based facilities for radio and optical astronomy, high-energy air showers, atmospheric Cherenkov, and neutrino and gravitational wave astronomy are involved with these attempts for correlated burst observations. Space-borne correlated observations of well-located gamma-ray bursts have also been attempted in the UV, EUV, and x-ray regions. A recent review of the present status of correlated gamma-ray burst observations is given by Schaefer (1994).

The long-baseline interplanetary network (IPN) of burst detectors have provided the most accurate locations for these correlated observations. With the loss of both the PVO 


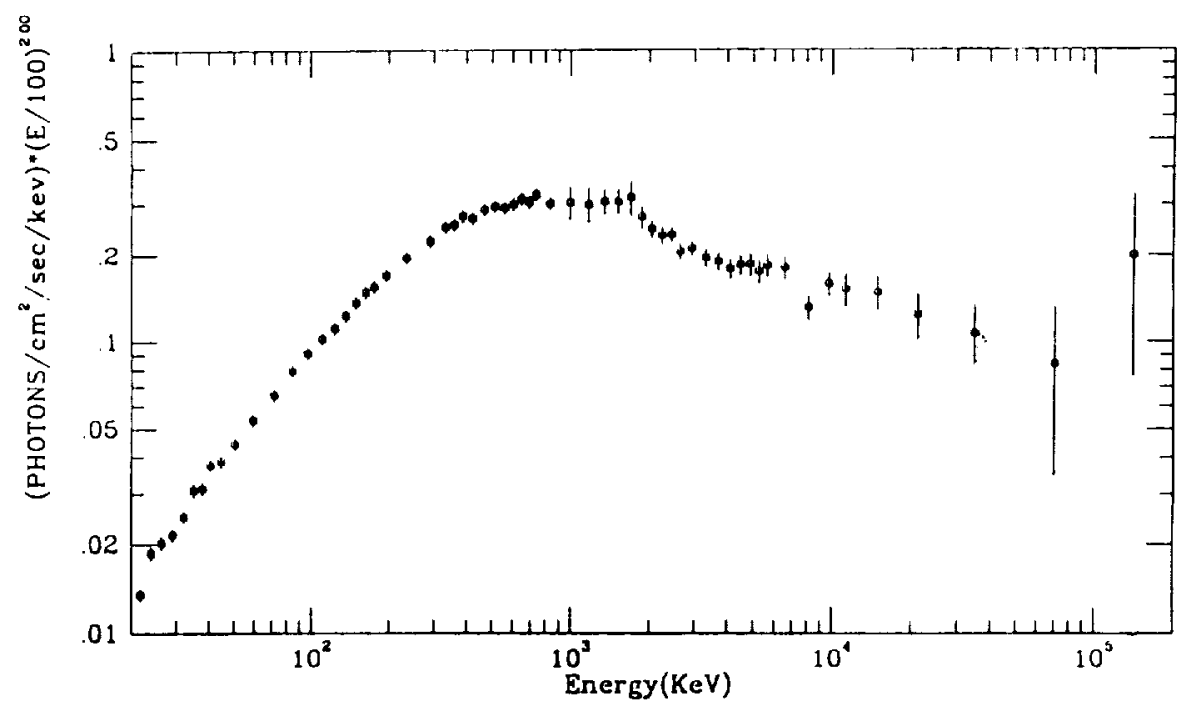

Figure 6. The spectrum of the strong burst GB 910503, measured by all four Compton Observatory experiments (Schaefer et al. 1994), extending from $20 \mathrm{keV}$ to 100 $\mathrm{MeV}$. The power spectrum is flat near $1 \mathrm{MeV}$.

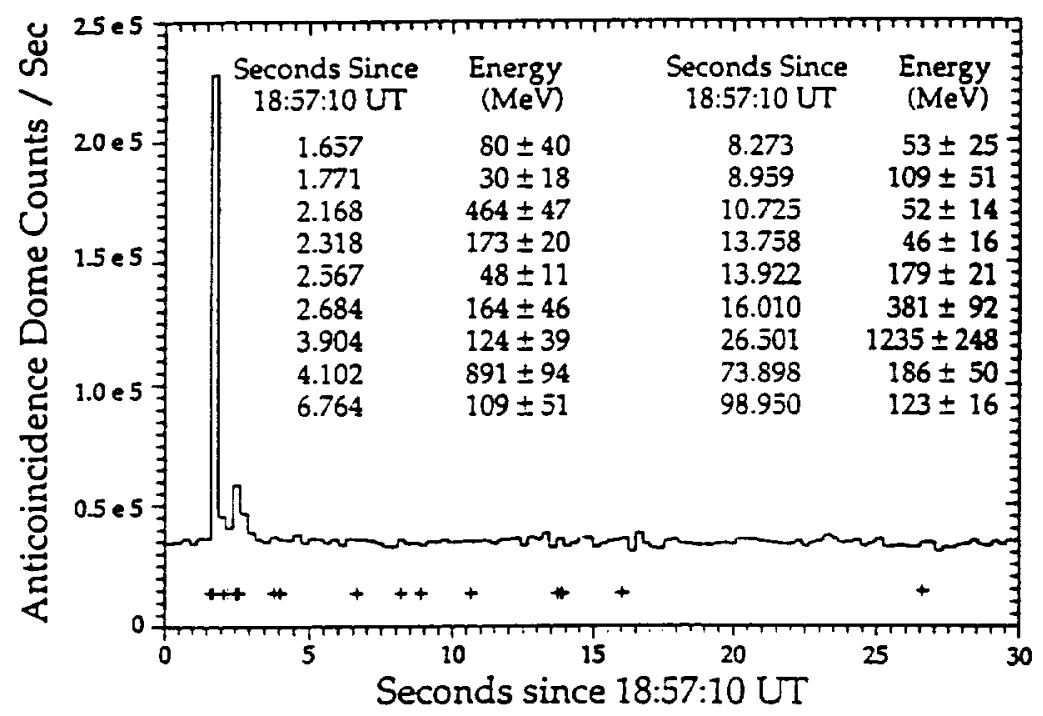

Figure 7. EGRET observations of GRB930131. This strong burst produced photons up to $1.2 \mathrm{GeV}$. The histogram shows the overall time profile of the burst as measured by the EGRET anticoincidence dome; the small crosses show the times of the high energy photons measured by the EGRET spark chamber. The times and energies of these photons are listed in the figure. 
and Mars Observer spacecraft in recent years, the detectors aboard the Ulysses spacecraft remain as the only long baseline component that can be used with earth-orbiting detectors. The narrow (usually a few arc-minutes) annuli derived from the Ulysses-Earth network observations can also be used with these coarser locations to greatly restrict the error box of some bursts. This situation will persist until the next Mars mission occurs, probably in 1996. For non-IPN localizations, the Compton Observatory instruments COMPTEL and EGRET can provide burst location accuracies of the order of one degree, for strong bursts which happen to be in their field-of-view. SIGMA-GRANAT and WATCH-GRANAT also continue to provide accurate locations for accessible bursts. Those ground-based searches that can utilize the rather coarse BATSE-derived burst locations ( 4 deg for intense bursts) such as wide field Schmidt cameras, can also respond to these bursts quicker than ever before possible.

BATSE has a quick alert capability that was developed to provide burst locations within several hours, under favorable conditions. A joint BATSE-COMPTEL capability also exists that is able to provide even more accurate $(\sim 1 \mathrm{deg})$ locations within several hours for those gamma-ray bursts which also happen to be within the COMPTEL field-of-view. This capability has been demonstrated already for the intense gamma-ray burst of 31 January 1993, when an extraordinary effort involving over 30 instruments observed the burst region within hours and days of its occurrence (Schaefer et al. 1994).

A new near-realtime BATSE burst location system called BACODINE (BAtse COordinates DIstribution NEtwork) (Barthelmy et al. 1994) is also underway. This system, when linked to a rapid slewing optical telescope, opens the exciting possibility of obtaining optical images of burst regions while the burst is in progress. Although images have been obtained previously with sky patrol plates, these have been very wide field instruments with relatively low sensitivity. A sensitive, wide-field transient optical camera has also been operating for over three years at Kitt Peak (Vanderspek et al. 1994).

Finally, several persons have suggested the possibility of measuring the absorption of soft $\mathrm{x}$-rays from bursts in the galactic plane as a means of determining their distance (cf. Schaefer 1994; Owens 1994).

\section{Burst Distributions}

The isotropy of the BATSE gamma-ray burst distribution, coupled with its inhomogeneity (as measured by the deficiency of weak gamma-ray bursts) continues to be the most surprising observation recent observation of gamma-ray bursts, and the one that has eliminated most of the expected and reasonable Galactic distribution models. Figure 9 shows the distribution of 921 BATSE gamma-ray bursts, plotted in Galactic coordinates. The BATSE sky exposure used in the derivation of this map is uniform to within $+/-20 \%$ (Fishman et al. 1994). When corrected for sky exposure, no significant dipole exists with respect to the Galactic center and there is no significant quadrupole moment with respect to the Galactic plane. (These anisotropies are also not present in the uncorrected data.) The inhomogeneity for the measurable bursts in this distribution, as measured by V/Vmax (cf. Schmidt 1968), is $\mathrm{V} / \mathrm{V} \max =0.32+1-0.01$. A value of 0.5 for $\mathrm{V} / \mathrm{V} \max$ is expected for a homogeneous distribution. The BATSE intensity distribution, measured with respect to 


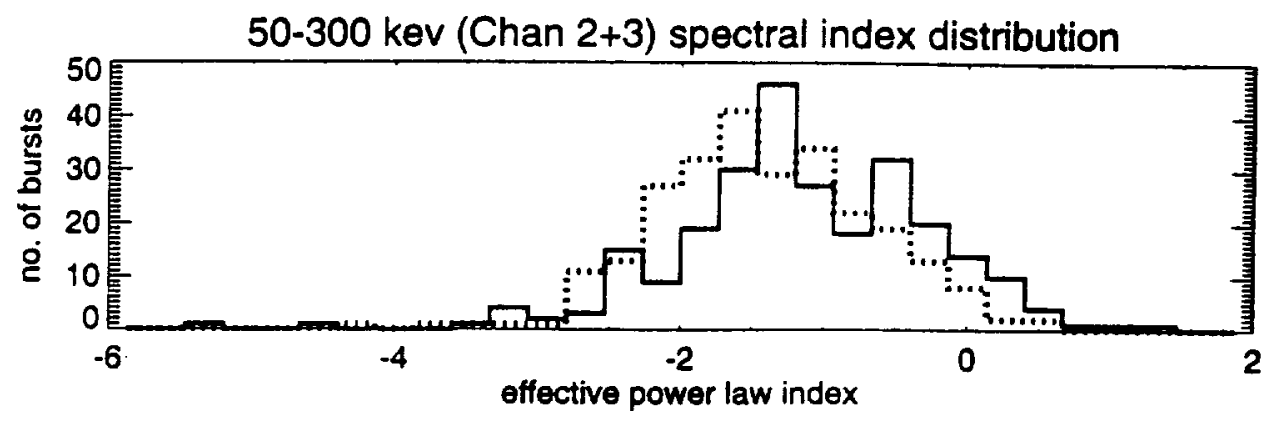

Figure 8. The distribution of power law spectral indices from a large number of gamma-ray bursts measured by the BATSE Large Area Detectors (LAD's). The solid histogram is the spectral fit at the peak flux of the burst; the dashed histogram is the fit to the integrated spectrum (Pendleton et al. 1994).

\section{BATSE Gamma-Ray Bursts}

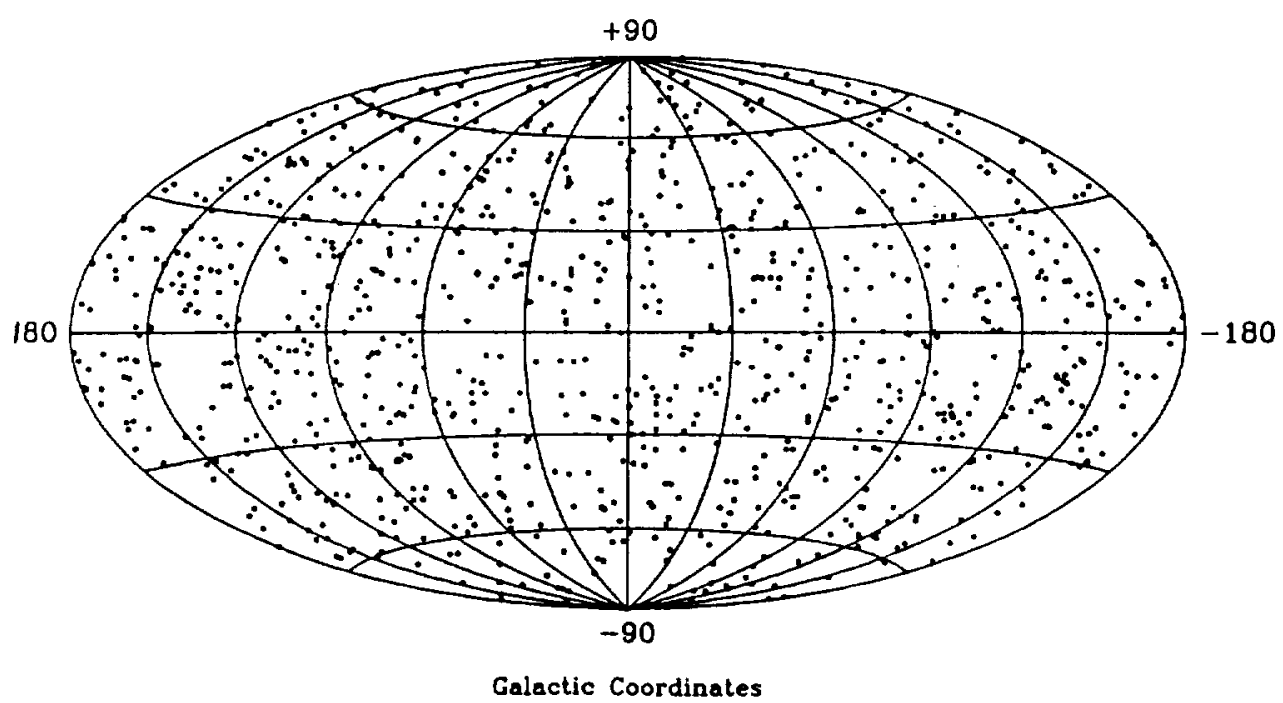

Figure 9. The distribution of 921 gamma ray bursts observed by BATSE on the Compton Gamma Ray Observatory. This preliminary map is plotted in Galactic coordinates. More accurate burst locations will become available as the data are further processed for subsequent catalogs. The isotropy of the bursts is apparent. 


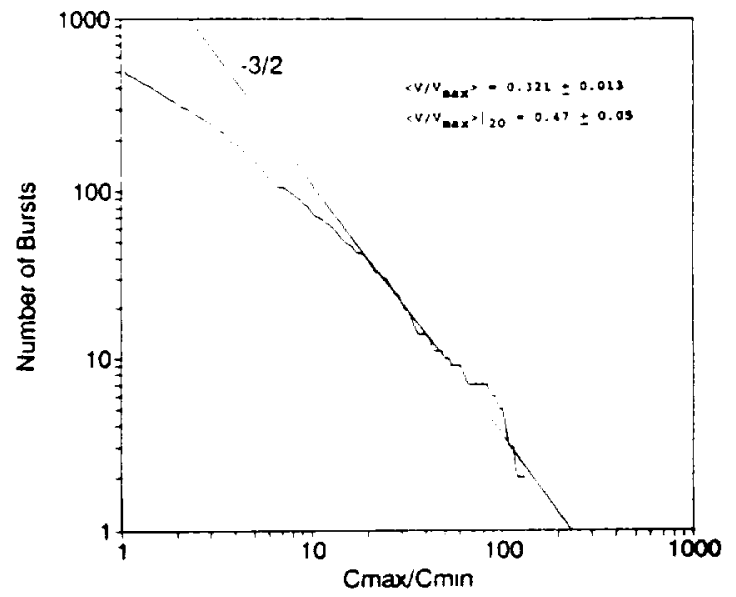

Figure 10. The intensity distribution of gamma-ray bursts measured by BATSE (from Meegan et al. 1994). In this integral plot, the intensity is plotted as a function of the instantaneous instrumental threshold. There is a clear deviation from a homogeneous distribution.

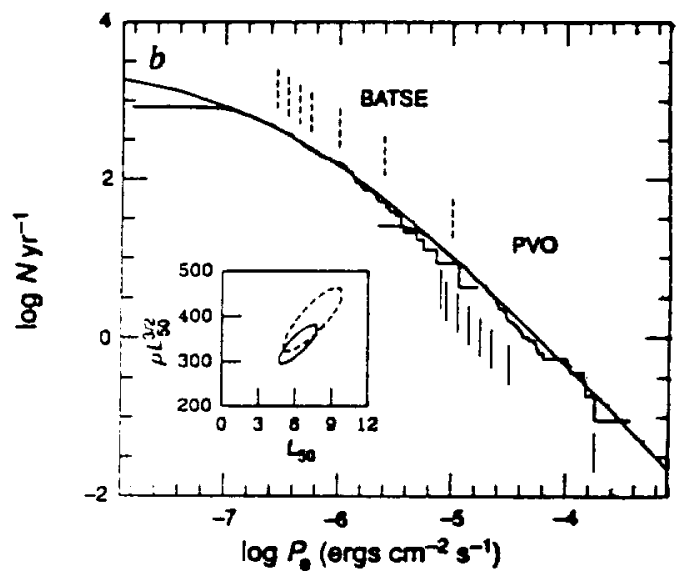

Figure 11. $\log N-\log P$ distribution of gamma-ray bursts from data combined from BATSE and Pioneer Venus Orbiter (PVO). The long duration of the PVO mission allowed better observations of the more rare, stonger events. The data from the two experiments fit well together and are seen to fit the expected $-3 / 2$ slope at the higher intensities. (see Fenimore et al. 1993 for further details of the plot) 
the instrumental threshold in shown in Figure 10 (from Meegan et al. 1994). An intensity distribution in peak flux units is given in Fishman et al. (1994). Recently, the BATSE intensity distribution has been combined with the PVO intensity distribution to yield a combined data set of almost four decades in intensity (Fenimore et al. 1993; Figure 11). This composite intensity distribution matches well in the overlap region, showing a smooth transition to the $-3 / 2$ power law expected at the higher intensities.

The three distribution models most often discussed as being compatible with the observation of isotropy and inhomogeneity are: 1) An extended solar system model such as an Oort cloud or other sun-centered large distribution (cf. White 1993; White 1994); 2) An extended Galactic halo or Galactic corona model, and 3) A cosmological model. However, a recent paper by Hakkila et al. (1994) shows that the parameter space available to the extended Galactic models is shrinking to unrealistic values, e.g. typical source distances of $\sim 70 \mathrm{kpc}$. Many believe that solar-system based models would likewise show an observable anisotropy. Thus, from the isotropy observations alone, one is increasingly forced to think in terms of cosmological models.

\section{Summary}

The gamma-ray burst enigma appears to be as great now as it was twenty years ago (Ruderman 1975). A wealth of new data on time profiles, spectral characteristics and burst distributions has thus far failed to provided conclusive evidence on the distance scale, central object(s) or emission mechanism(s) for the classical gamma-ray bursts. The isotropy and inhomogeneity of the bursts only shows that we are at the center of the apparent burst distribution. Many feel that the identification of a burst with an object in another wavelength region may be the key to understanding these objects. The recent EGRET-Compton Observatory discovery of delayed $\mathrm{GeV}$ emission from a burst is yet another severe constraint for many of the burst models. The field continues to be exciting and frustrating 


\section{REFERENCES}

Atteia, J.-L., these proceedings (1994).

Band, D. et al., Ap. J. 413, 281 (1993).

Band, D. et al, Ap. J., in press (1994).

Barthelmy, S. et al., Huntsville Gamma-ray Burst Workshop, AIP Conf. Proc. \#307 (AIP:New York) in press (1994).

Bhat, P.N. et al., Nature 359, 219 (1992).

Brock, M. N. et al., Huntsville Gamma-ray Burst Workshop, AIP Conf. Proc. \#307 (AIP:New York) in press (1994).

Dingus, B.L. et al., Huntsville Gamma-ray Burst Workshop, AIP Conf. Proc. \#307 (AIP:New York) in press (1994).

Fenimore, E.E. et al., Nature 366, 40 (1993).

Fishman, G.J. et al., Ap. J. Supp. 92,229 (1994).

Fishman, G.J., Brainerd, J.J. and Hurley, K., eds., Huntsville Gamma-ray Burst Workshop, AIP Conf. Proc. \#307 (AIP:New York) in press (1994).

Ford, L. et al., Huntsville Gamma-ray Burst Workshop, AIP Conf. Proc. \#307 (AIP:New York) in press (1994)

Friedlander, M., Gehrels, N. and Macomb, D, eds., Compton Gamma Ray Observatory, AIP Conf. Proc. \#280 (AIP:New York) (1993).

Hakkila, J. et al., Ap. J. 422, 659 (1994).

Hanlon, L.O. et al., Huntsville Gamma-ray Burst Workshop, AIP Conf. Proc. \#307 (AIP:New York) in press (1994).

Hartmann, D., these proceedings (1994).

Hurley, K., Huntsville Gamma-ray Burst Workshop, AIP Conf. Proc. \#307 (AIP:New York) in press (1994).

Hurley, K. and Cline, T, Huntsville Gamma-ray Burst Workshop, AIP Conf. Proc. \#307 (AIP:New York) in press (1994).

Kouveliotou, C. et al., Ap. J. Lett. 413, L101 (1993).

Kouveliotou, C. et al., Nature 368, 125 (1994).

Kulkarni, S.R. et al., Nature 368, 129 (1994).

Lestrade, J.P. et al., Huntsville Gamma-ray Burst Workshop, AIP Conf. Proc. \#307 (AIP:New York) in press (1994).

Lestrade, J.P., Ap. J. Lett., in press (1994).

Link, B., Epstein, R.I. and Priedhorsky, W.C., Ap. J. Lett. 408, L81 (1993).

Meredith, D. et al., Huntsville Gamma-ray Burst Workshop, AIP Conf. Proc. \#307 (AIP:New York) in press (1994).

Murakami, T, et al., Nature 368, 127 (1994).

Nemiroff, R.J. et al., Ap. J. 423, 432 (1994).

Nemiroff, R.J., Huntsville Gamma-ray Burst Workshop, AIP Conf. Proc. \#307

(AIP:New York) in press (1994); also Comments in Astrophys. (1994). 
Owens, A et al. Huntsville Gamma-ray Burst Workshop, AIP Conf. Proc. \#307 (AIP:New York) in press (1994).

Paciesas, W.S. and Fishman, G.J., eds., Huntsville Gamma-ray Burst Workshop, AIP Conf. Proc. \#265 (AIP:New York) (1992).

Pendleton, G.N. et al., Ap. J., submitted (1994)

Pendleton, G.N. et al., Huntsville Gamma-ray Burst Workshop, AIP Conf. Proc. \#307

(AIP:New York) in press (1994).

Norris, J. et al., Ap. J. 424, 540 (1994).

Ruderman, M., Ann. N.Y. Acad. Sci. 262, 164 (1975).

Schaefer, B.E. et al., Ap. J. Lett. 393, L51 (1992).

Schaefer, B.E. et al., Ap. J. Lett. 422, L71 (1994).

Schaefer, B.E., Huntsville Gamma-ray Burst Workshop, AIP Conf. Proc. \#307 (AIP:New York) in press (1994).

Schaefer, B.E. et al., Ap. J. Supp. 92, 285 (1994).

Schmidt, M., Ap. J. 151, 393 (1968).

Share, G. et al., Adv. Sp. Res. 6, 15 (1988).

Share, G. et al., Huntsville Gamma-ray Burst Workshop, AIP Conf. Proc. \#307 (AIP:New York) in press (1994)

Vanderspek, R., Krimm, H., and Ricker, G., Huntsville Gamma-ray Burst Workshop, AIP Conf. Proc. \#307 (AIP New York) in press (1994).

Yoshida, A et al., Pub. Astron. Soc. Japan 41, 509 (1989)

White, R.S., Huntsville Gamma-ray Burst Workshop, AIP Conf. Proc. \#307

(AIP:New York) in press (1994).

White, R.S., Ap. and Sp. Sci. 208, 301 (1993) 